\title{
Evaluation of HO-1 expression, cellular ROS production, cellular proliferation and cellular apoptosis in human esophageal squamous cell carcinoma tumors and cell lines
}

\author{
QUAN-GUANG REN，SHENG-LI YANG，JIAN-LI HU，PIN-DONG LI，YE-SHAN CHEN and QIU-SHUANG WANG \\ Cancer Center, Union Hospital, Tongji Medical College, Huazhong University of Science and Technology, \\ Jianghan, Wuhan, Hubei 430022, P.R. China
}

Received October 18, 2015; Accepted November 24, 2015

DOI: $10.3892 /$ or.2016.4556

\begin{abstract}
Patients with esophageal squamous cell carcinoma (ESCC) have a poor prognosis. However, the related mechanisms are unclear, thus we investigated the expression of HO-1 in ESCC tissue and explored possible mechanisms of tumor progression. Expression of HO-1 was examined by immunohistochemistry in 143 ESCC tumors. The correlation of HO-1 with clinicopathological characteristics was also examined. Two human ESCC cell lines, TE-13 and Eca109 were studied. Silencing of cell line HO-1 by specific small interfering RNA (siRNA) was evaluated using real-time quantitative PCR. Cell line viability, apoptosis and intracellular levels of reactive oxygen species (ROS) after transfection were determined using MTT and flow cytometry, respectively. HO-1, Bax, Bcl-2 and A-caspase-3/-9 expression was evaluated using western blot analyses. We found that $\mathrm{HO}-1$ was expressed in 58 of 143 ESCC tumors, mainly in the cytoplasm. There was a significant association between HO-1 expression and tumor grade $(\mathrm{P}<0.001)$. Knockdown of HO-1 expression in cell lines was associated with significantly decreased cellular proliferation $(\mathrm{P}<0.05)$ and a higher rate of apoptosis $(\mathrm{P}<0.001) 48 \mathrm{~h}$ after treatment. Treatment of the cell lines with the ROS inhibitor $\mathrm{N}$-acetylcysteine abrogated this effect. Knockdown of HO-1 was associated with increased A-caspase-3 and -9 expression, but no change in Bax or $\mathrm{Bcl}-2$ expression or $\mathrm{Bax} / \mathrm{Bcl}-2$ ratio was observed. Thus, the present study identified that ESCC tumors frequently expressed HO-1. Knockdown of HO-1 promoted apoptosis through activation of a ROS-mediated caspase apoptosis pathway.
\end{abstract}

Correspondence to: Professor Jian-Li Hu, Cancer Center, Union Hospital, Tongji Medical College, Huazhong University of Science and Technology, 1277 Jiefangdadao, Jianghan, Wuhan, Hubei 430022, P.R. China

E-mail: j15199@126.com

Key words: heme oxygenase-1, reactive oxygen species, esophagus, squamous cell carcinoma, apoptosis

\section{Introduction}

Esophageal squamous cell carcinoma (ESCC) is a malignancy associated with high mortality in China. Multidisciplinary treatment (surgery, radiotherapy and chemotherapy) is generally used to treat locally advanced and metastatic ESCC. Unfortunately ESCC is frequently resistant to radiotherapy and chemotherapy. The 5-year survival of ESCC patients is only $20.9 \%$ (1). Better understanding of prognostic indicators in ESCC is needed.

Heme oxygenase-1 (HO-1) is a stress-induced gene with anti-inflammatory, anti-apoptosis, antioxidation and drug resistance inducing properties. The expression of HO-1 in normal human tissues is extremely low. Increased expression of HO-1 has been seen after exposure to alcohol and spicy foods. Increased HO-1 expression has been reported in a variety of tumors and has been associated with ESCC tumor invasion, metastases, chemotherapy-induced apoptosis and worse patient prognosis (2,3). HO-1 expression is thought to be regulated by upstream expression of nuclear-related factor 2 (Nrf-2). Cell growth is inhibited with inhibition of HO-1 and increased Nrf-2 expression (4). Increased HO-1 expression is also associated with increased removal of reactive oxygen species (ROS) and maintenance of the internal cellular environment (5).

We previously reported that overexpression of HO-1 can significantly impede the apoptosis of ESCC cells (6). It is not known whether decreasing HO-1 expression induces apoptosis or impact ROS removal. In order to better understand HO-1 control, we evaluated the expression of HO- 1 , HIF-1 $\alpha$ and EGFR protein in human ESCC tissue using immunochemistry. We also evaluated intracellular ROS levels and apoptosis-related HO-1 protein levels in ESCC cell lines.

\section{Materials and methods}

Patients and tissue specimens. Medical records at the Union Hospital Affiliated to Huazhong University of Science and Technology were reviewed for patients with ESCC. All patients had histologic confirmation of their diagnosis. Clinical features were collected including patient age, clinical stage, tumor grade, presence of mediastinal lymph node metastases. Tumor blocks were obtained for immunohistochemical evaluation of 
HO-1, HIF-1 $\alpha$ and EGFR expression in the tumor. Clinical staging was performed using WHO 2003 AJCC Sixth Edition PTNM staging criteria. No patient received chemotherapy or radiotherapy before biopsy or surgical resection. The use of ESCC specimens was approved by the local Ethics Committee. All patients have given their informed consent for the present study.

Immunohistochemical staining. Tumor blocks were obtained from the Department of Pathology and immunohistochemical staining was performed as previously described (7). The protein expression of HO-1, HIF- $1 \alpha$ and EGFR protein were scored according to the number of cells exhibiting cytoplasmic and nuclear staining using the following classification system: I, no staining; II, nuclear staining in $10 \%$ of cells and/or weak cytoplasmic staining; III, nuclear staining in $10-50 \%$ of cells and/or distinct cytoplasmic staining; IV, nuclear staining in 50\% of cells and/or strong cytoplasmic staining. Tumors with I or II amounts of staining were considered negative for expression and tumors with III or IV were considered positive.

Cell culture and transfection with $\mathrm{HO}-1$ small interfering $R N A$ (siRNA). The human ESCC cell lines TE-13 and Eca109 were a gift from the He Bei, Medical University Affiliated Cancer Hospital. HO-1-siRNA and the empty vector containing a nonsense RNA sequence were purchased from Guangzhou RiboBio Co., Ltd., Guangzhou, China. The RNA transfection kit was from Guangzhou RiboBio Co, Ltd. TE-13 and Eca109 were cultured in Dulbecco's modified Eagle's medium (DMEM) median containing 10\% fetal bovine serum. The two cell lines were maintained in a humidified incubator at $37^{\circ} \mathrm{C}$ in a $5 \% \mathrm{CO}_{2}$ atmosphere. Four experimental groups were examined including negative untreated cell line controls, cell line controls transfected with a nonsense RNA sequence, cell lines transfected with si-HO-1 and cell lines transfected with si-HO-1-NAC (a powerful antioxidant, NAC namely $\mathrm{N}$-acetyl cysteine). Transfection was performed using $100 \mathrm{nM}$ siRNA. Logarithmic growth phase cells were harvested and plated at a density of $2 \times 10^{5}$ cells/well in 6-well plates. Cells were grown and the expression of HO-1 in transfected cells quantified using real-time PCR.

RNA isolation and reverse-transcription PCR. Cell lines were harvested at different time points after transfection. Total RNA was extracted from cell lines using TRIzol reagent (Invitrogen). The purity and concentration of RNA was determined using a NanoDrop Spectrophotometer (ND-2000; NanoDrop Technology, Wilmington, DE, USA). cDNA was produced using a reverse-transcription kit (Takara Bio, Inc.). Real-time quantitative PCR was performed using the SYBRGreen Prime Script RT-PCR kit (Takara Bio, Inc.) and the Real-time PCR detection system (Applied Biosystems). GAPDH was used as an internal control. The primers for HO-1 were: forward, GTCAGGCAGAGGGTGATAGAAG and reverse, GTGTAAGGACCCATCGGAGAAG. The primers for GAPDH were: forward, TCCCATCACCATCTTCCAG and reverse, GAGCCCCAGCCTTCTCCAT. The results of three independent experiments were analyzed using the $2^{-\Delta \Delta \mathrm{Ct}}$ method.
MTT assay. TE-13 and Eca109 cells were seeded into 96-well plates at a density of $8 \times 10^{3}$ cells/well, in a volume of $180 \mu 1$ culture medium. The four previously described treatment groups were evaluated. Cells in each group were cultured to $70 \%$ confluency before transfection. MTT $(20 \mu \mathrm{l})$ was then added to each well after $24 \mathrm{~h}, 48 \mathrm{~h}$ and $72 \mathrm{~h}$ of incubation and incubated for $4 \mathrm{~h}$. The media were then removed and $150 \mu \mathrm{l}$ dimethylsulfoxide (DMSO) added to each well. The incubation plates were gently mixed for $10 \mathrm{~min}$ before viability analysis. Cell viability was determined as absorbance at $490 \mathrm{~nm}$ at $24 \mathrm{~h}$, $48 \mathrm{~h}$ and $72 \mathrm{~h}$ after MTT treatment. Four wells of each cell line were evaluated for each experiment. The results of three independent experiments are reported.

Flow cytometric analysis. TE-13 and Eca109 cells were inoculated into 6 -well plates at a density of $2 \times 10^{5}$ cells/well. Procedures of interfering HO-1 in TE-13 and Eca109 cells were executed according to manual of infection reagent kit. Flow cytometric analysis was performed as previously described (6). The results of three independent experiments were reported.

Western blot analysis. HO-1 interference in two cell lines was treated with transfection reagent kit represented above. Protein lysates were obtained $48 \mathrm{~h}$ after transfection. Protein $(100 \mu \mathrm{g})$ was loaded into each gel lane prior to electrophoresis on $12 \%$ SDS polyacrylamide gels. Electrophoresed protein was transferred to polyvinylidene defluoride (PVDF) membranes. The membranes were blocked using 5\% skimmed milk at room temperature for $1.5 \mathrm{~h}$, and then incubated at $4^{\circ} \mathrm{C}$ overnight with primary antibodies directed against HO-1, Bax, Bcl-2, A-caspase-3/-9 or $\beta$-actin (1:500 dilution; Abnova Corporation, Taipei City, Taiwan). Peroxidase-conjugated secondary antibodies were used to visualize the primary antibodies with an enhanced chemiluminescence reagent (Beyotime). Protein expression was quantitated using densitometry analysis and normalized against $\beta$-actin.

Statistical analysis. SPSS 17.0 software (SPSS, Inc., Chicago, IL, USA) was used to evaluate data. Data are reported as the mean \pm standard deviation (SD). Statistical significance was determined using a two-sided, unpaired t-test. P-value $<0.05$ was considered to indicate a statistically significant result.

\section{Results}

In total, 143 male patients with ESCC were identified at the Union Hospital Affiliated to Huazhong University of Science and Technology between April 2006 and October 2007. The mean patient age was $59.5 \pm 1.4$ years old (range, $40-83$ years old).

Protein expression of HO-1, HIF-l $\alpha$ and EGFR in human ESCC tumors. Immunohistochemical probing of fixed resected ESCC tissues was performed. HO-1 was expressed in 58 of $143(40.6 \%)$ patient tumors. The HO-1 protein was mainly localized to the cytoplasm of tumor cells (Fig. 1A). HIF-1 $\alpha$ expression was observed in $43.4 \%$ (62/143) of patient tumors, EGFR in 58\% (83/143). HIF-1 $\alpha$ was mainly located in the nucleus and EGFR was mainly localized in cytoplasm (Fig. 1B and C). 
A

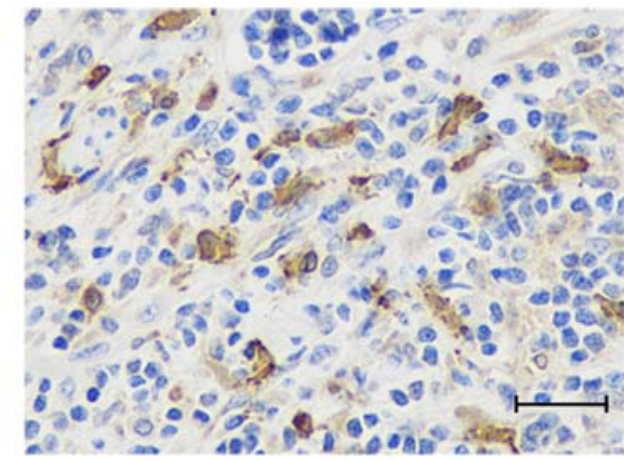

C

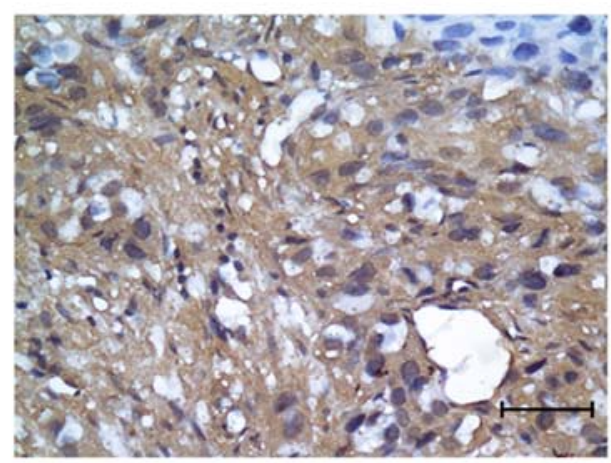

B

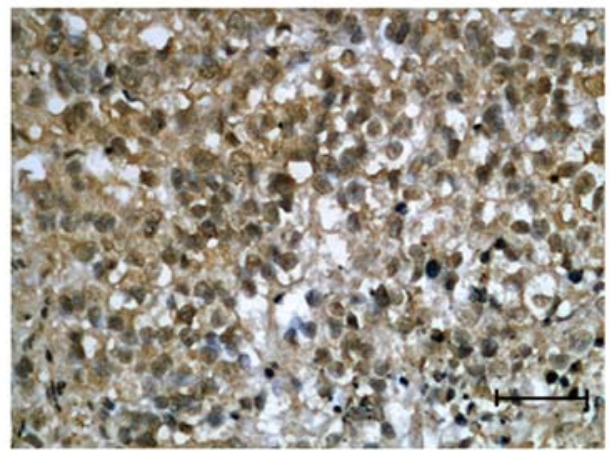

Figure 1. Immunohistochemical staining of heme oxygenase-1 (HO-1) hypoxia inducible factor $1-\alpha(\mathrm{HIF}-1 \alpha)$ and epidermal growth factor receptor (EGFR). (A) HO-1 displays cytoplasmic staining in this representative specimen of esophageal squamous cell carcinoma (ESCC) tissue. (B) HIF-1 $\alpha$ displays mainly nuclear staining in this representative specimen of ESCC tissue. (C) EGFR displays mainly cytoplasmic staining in this representative specimen of ESCC tissue. Scale bar, $50 \mu \mathrm{m}$.
Table I. Relationship between HO-1 expression and HIF-1 $\alpha$, EGFR expression in ESCC tumors ( $\mathrm{N}=143)$.

\begin{tabular}{lcrrr}
\hline & \multicolumn{2}{c}{ HO-1 expression } & & \\
\cline { 2 - 3 } Related gene & Negative & Positive & $\chi^{2}$ & P-value \\
\hline HIF-1 $\alpha$ & & & & \\
$\quad$ Negative & 66 & 15 & 37.646 & $\mathbf{< 0 . 0 0 1}$ \\
Positive & 19 & 43 & & \\
EGFR & & & & \\
Negative & 44 & 16 & 10.914 & $\mathbf{< 0 . 0 0 1}$ \\
Positive & 41 & 42 & & \\
\hline
\end{tabular}

HO-1, heme oxygenase 1; HIF-1 $\alpha$, hypoxia inducible factor $1-\alpha$; EGFR, epidermal growth factor receptor; ESCC, esophageal squamous cell carcinoma. Bold type, statistically significant.

Correlation between HO-1 expression and HIF-1 $\alpha, E G F R$ expression in ESCC tumors. HO-1 expression was positively correlated with HIF- $1 \alpha$ and EGFR expression in the 143 ESCC tumors $(\mathrm{P}<0.001$ for both, Table I).

Correlation between HO-1 expression in ESCC tumors and clinicopathological characteristics. There was no association between HO-1 expression and patient clinical stage $(\mathrm{P}=0.641)$ or age $(\mathrm{P}=0.409)$ (Table II). Increasing tumor histologic grade was associated with increasing expression of HO-1 ( $\mathrm{P}=0.001)$. There was no correlation between HO-1 expression and mediastinal lymph node metastases $(\mathrm{P}=0.415)$.

Effect of transfection with HO-1 siRNA on HO-1 mRNA and protein expression in TE-13 and Ecal09 cell lines. Real-time quantitative PCR (Fig. 2A) and western blotting (Fig. 2B)
Table II. Correlation between HO-1 and clinicopathological characteristics.

\begin{tabular}{lcccc}
\hline & & \multicolumn{3}{c}{ HO-1 } \\
\cline { 3 - 5 } $\begin{array}{l}\text { Clinicopathological } \\
\text { characteristics }\end{array}$ & & $\begin{array}{c}\text { Positive rate } \\
(\%)\end{array}$ & $\chi^{2}$ & P-value \\
\hline $\begin{array}{l}\text { Age (years) } \\
<60\end{array}$ & 65 & $25(38.5)$ & 0.218 & 0.641 \\
$\geq 60$ & 78 & $33(42.3)$ & & \\
Clinical stage & & & & \\
II & 39 & $18(46.2)$ & & \\
III & 53 & $23(43.5)$ & 1.788 & 0.409 \\
IV & 51 & $17(33.3)$ & & \\
Grade & & & & \\
G1 & 32 & $9(28.1)$ & & \\
G2 & 50 & $13(26.0)$ & 15.068 & $\mathbf{0 . 0 0 1}$ \\
G3 & 61 & $36(59.0)$ & & \\
Mediastinal lymph & & & & \\
node metastasis & & & & \\
Yes & 73 & $32(43.8)$ & 0.664 & 0.415 \\
No & 70 & $26(37.1)$ & & \\
\hline
\end{tabular}

HO-1, heme oxygenase 1; MLNM, mediastinal lymph node metastasis. Bold type, statistically significant.

demonstrated decreased expression of HO-1 in the TE-13 and Eca109 cell lines. There was no change in expression of the untransfected control cell lines or the cell lines transfected with nonsense mRNA. Significantly less HO-1 expression 
A

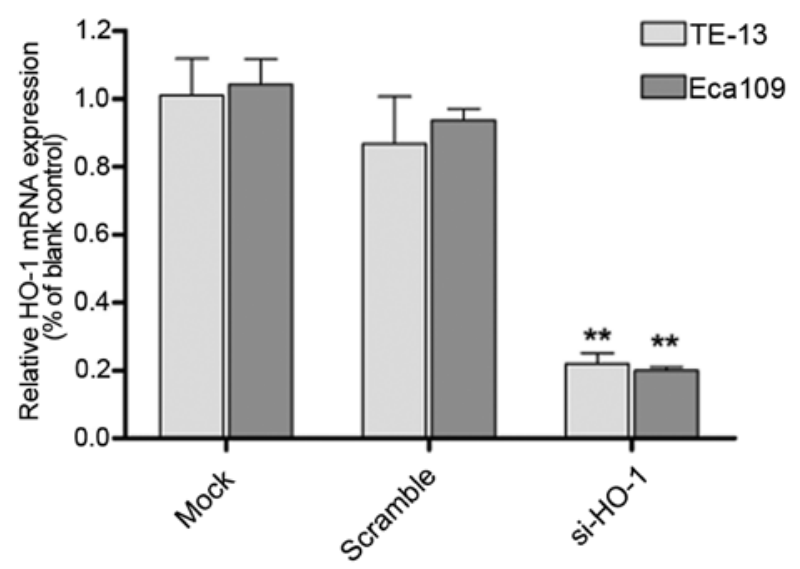

B

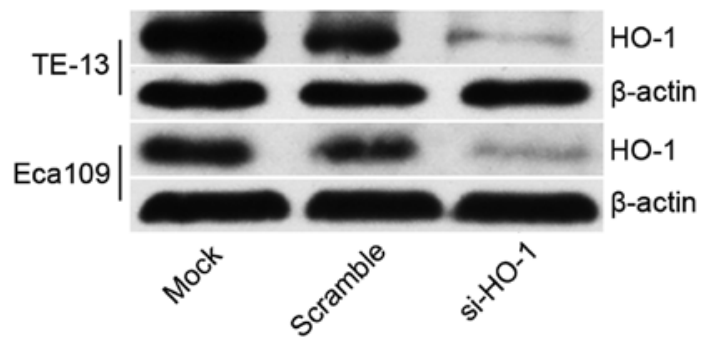

Figure 2. The effect on HO-1 expression after HO-1 small interfering RNA transfection in TE-13 and Eca109 cell lines. Both TE-13 and Eca109 were transiently transfected with HO-1 special small interfering RNA after incubation for $48 \mathrm{~h}$. Untransfected cell lines and cell lines transfected with nonsense RNA were used as controls. (A) Real-time quantitative polymerase chain reaction demonstrated decreased expression of HO-1 after transfection with HO-1 small interfering RNA, compared to the scramble group ( $\left.{ }^{* *} \mathrm{P}<0.01\right)$. (B) $\mathrm{HO}-1$ protein expression was similarly decreased on western blot analyses $\left({ }^{* *} \mathrm{P}<0.01\right)$.

was seen in the HO-1 siRNA transfected cell lines $(\mathrm{P}<0.01$ for both cell lines).

Effect of blocking HO-1 expression on cell line viability. MTT assays were performed to assess cell viability. All four treatment groups had decreased cell viability at $24 \mathrm{~h}, 48 \mathrm{~h}$ and $72 \mathrm{~h}$ in TE-13 (Fig. 3A) and Eca109 cell line (Fig. 3B) after treatment, compared to their respective controls. The lowest cell viability was observed at $48 \mathrm{~h}$. si-HO-1-treated cells had less cell viability at each time point, compared to untransfected cells and cells transfected with nonsense RNA $(\mathrm{P}<0.05)$. The cell viability of si-HO-1-NAC-treated cells was similar to that of the two control groups $(\mathrm{P}>0.05)$.

Effect of blocking HO-1 expression on cell line apoptosis. Flow cytometry after $48 \mathrm{~h}$ treatment showed the apoptosis rate of untransfected cells transfected with nonsense RNA, cells transfected with si-HO-1 and cells transfected with si-HO-1-NAC was $(3.8 \pm 1.2) \%,(6.8 \pm 1.9) \%,(27.4 \pm 1.6) \%$ and $(4.1 \pm 1.5) \%$, respectively (Fig. 4A) in the TE-13 cell line. The apoptosis rate in the same treatment groups was $(2.6 \pm 1.0) \%$, $(5.5 \pm 1.6) \%,(24.2 \pm 2.1) \%$ and $(3.9 \pm 1.7) \%$, respectively (Fig. 4B) in the Eca109 cell line. The rate of apoptosis was greatest in the si-HO-1-treated cell lines $(\mathrm{P}<0.001)$ compared to the two control treatment groups in both cell lines. NAC had a protective effect in cells transfected with si-HO-1 $(\mathrm{P}<0.001)$.
A

TE-13

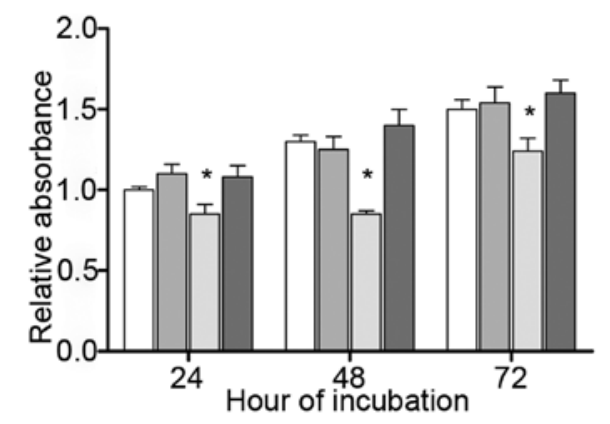

$\square$ Mock

$\square$ Scramble

$\square$ si-HO-1

$\square$ si-HO-1-NAC

B

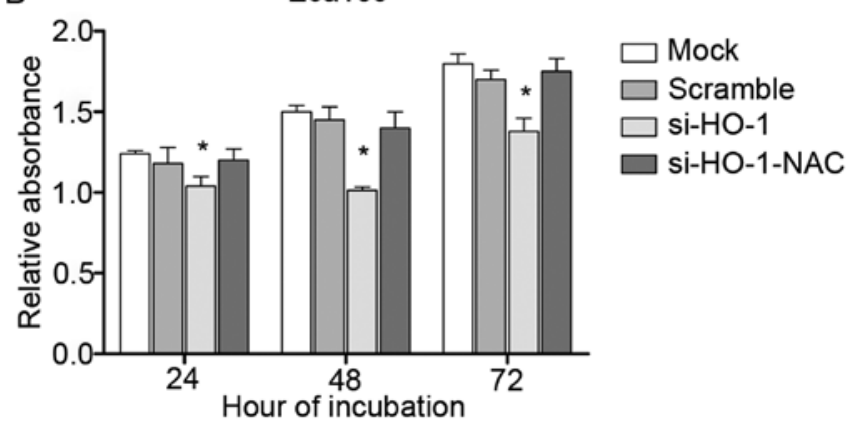

Figure 3. Cell viability in transfected TE-13 and Eca109 cells. Inhibition of HO-1 expression decreased the viability of ESCC cell lines as measured by MTT assay. (A) TE-13 cell line viability was inhibited at 24, 48 and $72 \mathrm{~h}$ after cell treatments. The greatest inhibition was seen at $48 \mathrm{~h}$ with TE-13 cells ( $\mathrm{P}<0.05$, compared with the scramble). (B) Eca109 cell line proliferation was inhibited at 24,48 and $72 \mathrm{~h}$ after treatment. The greatest inhibition was shown at $48 \mathrm{~h}\left({ }^{*} \mathrm{P}<0.05\right.$, compared with the scramble).

Effect of blocking HO-1 expression on cell line intracellular ROS levels. Mean fluorescent intensity (MFI) of intracellular ROS in all four cell line treatment groups was evaluated at $48 \mathrm{~h}$ using flow cytometry. The MFI of the untreated cell line controls, cell line controls transfected with nonsense RNA, cell lines transfected with si-HO-1 and cell lines transfected with si-HO-1-NAC was $98.1 \pm 4.7,99.4 \pm 5.2,360.5 \pm 8.8$ and 105.1 \pm 4.0, respectively, in TE-13 cells (Fig. 5A) and 95.3 \pm 3.9 , 103.7 $\pm 6.3,350.1 \pm 7.2$ and 101.4 \pm 5.7 , respectively, in Eca109 cells (Fig. 5B). The MFI of the si-HO-1 transfected cell lines were significantly greater than that of the two control groups $(\mathrm{P}<0.001$ for both cell lines). The MFI of the si-HO-1-NAC transfected group was significantly less than that of the si-HO-1 transfected cell lines ( $\mathrm{P}<0.001$ for both cell lines).

Effect of blocking HO-1 expression on cell line protein expression of Bax, Bcl-2, A-caspase-3 and -9. Protein expression was evaluated using western blot analyses of cell line protein isolated $48 \mathrm{~h}$ after treatment. There was no statistical difference in Bax or Bcl-2 expression of cell lines transfected with si-HO-1 or si-HO-1-NAC, compared to control (scramble). There was no statistical difference in the $\mathrm{Bax} / \mathrm{Bcl}-2$ expression ratio of cell lines transfected with si-HO-1 or si-HO-1-NAC.

Expression of A-caspase-3 and -9 was increased in the si-HO-1 group, compared to the two control groups $(\mathrm{P}<0.05$ for both groups). The si-HO-1-NAC transfected cell lines had significantly less expression of A-caspase- 3 and -9 than the si-HO-1 transfected cell lines $(\mathrm{P}<0.05)$ (Fig. 6). 
A

$\bar{a}$
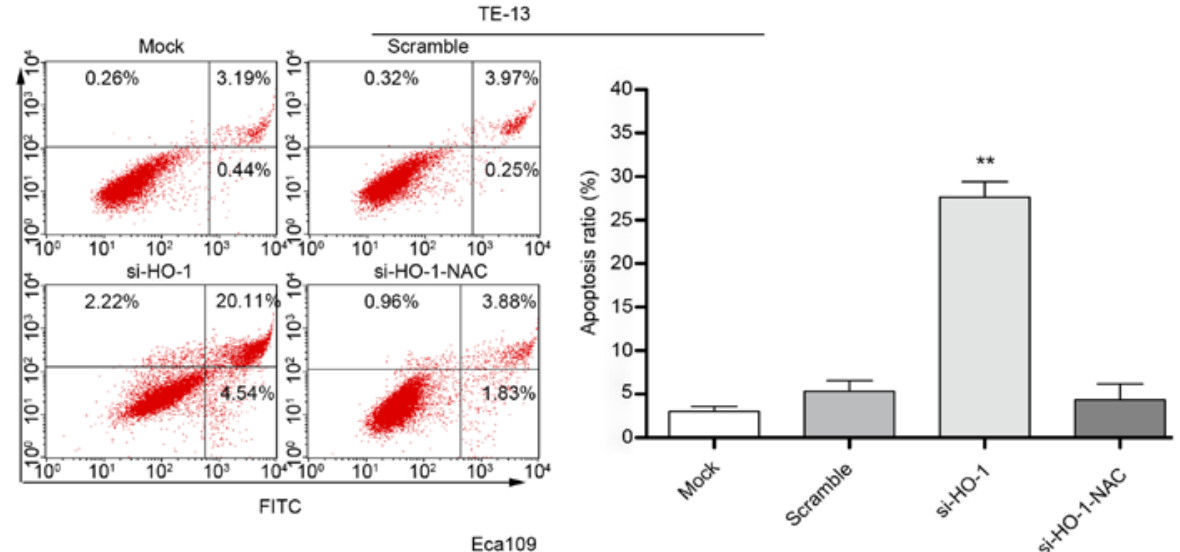

B
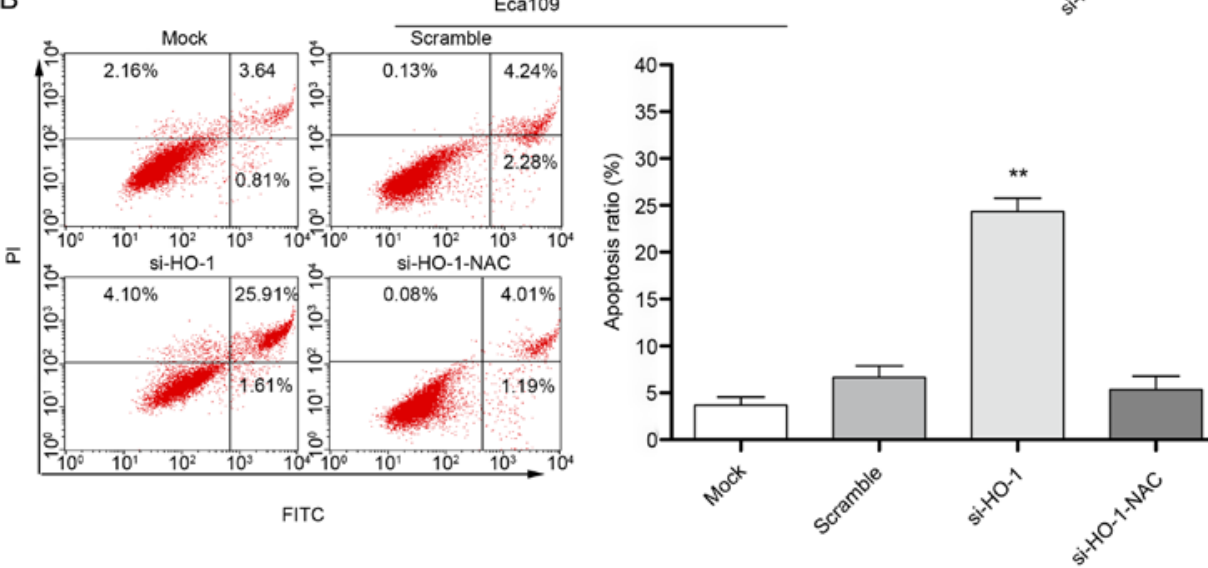

Figure 4. Cell apoptosis in TE-13 and Eca109 cells with decreased HO-1 expression. Flow cytometry demonstrated increased apoptosis in cell lines with decreased HO-1 expression. (A) The rate of apoptosis in TE-13 cells was increased $48 \mathrm{~h}$ after transfection with si-HO-1 (" $\mathrm{P}<0.001$, compared with the Scramble). (B) The rate of apoptosis in Eca109 cells was increased $48 \mathrm{~h}$ after transfection with si-HO-1 ( ${ }^{* *} \mathrm{P}<0.001$, compared with the scramble).

A

TE-13

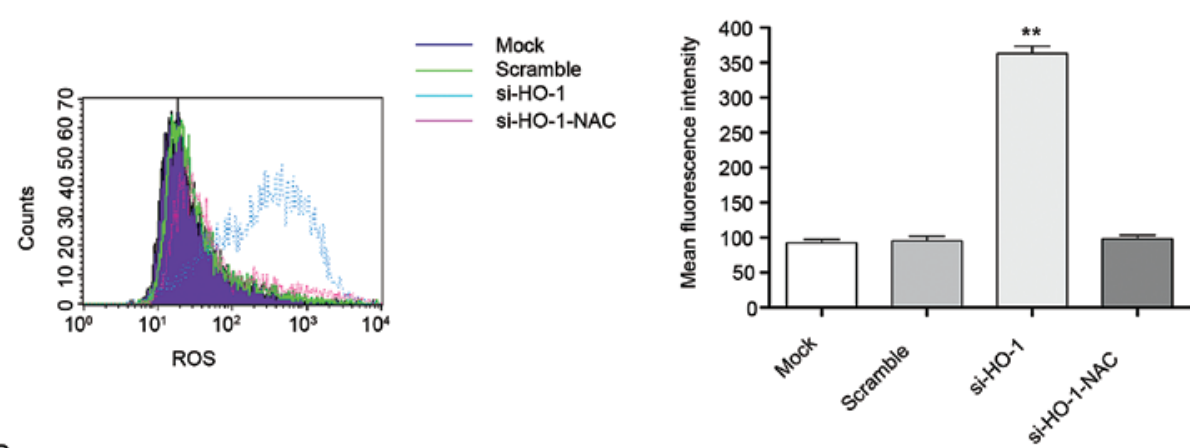

B

Eca109
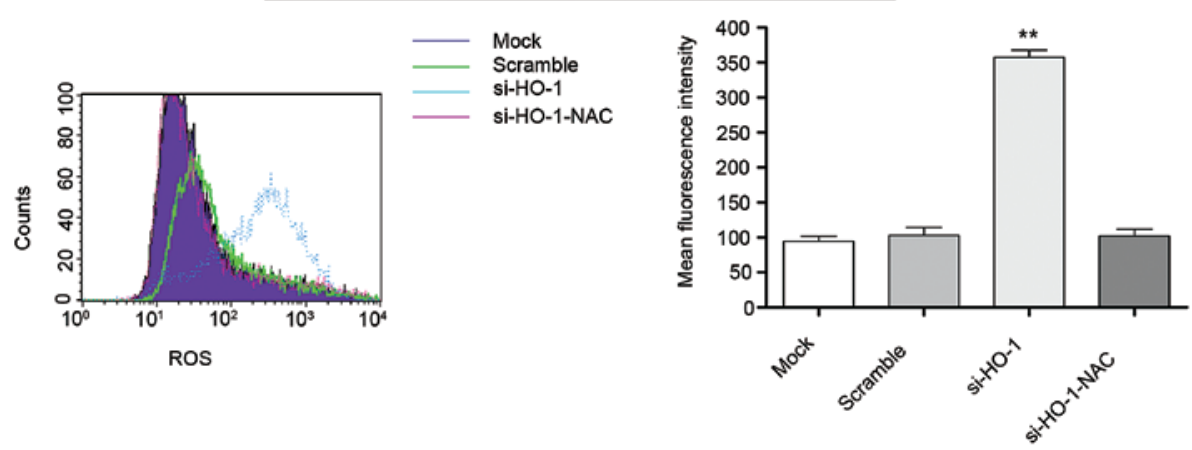

Figure 5. Intracellular ROS expression in TE-13 and Eca109 cell lines. Decreased HO-1 expression was associated with increased mean fluorescence intensity (MFI) of intracellular ROS in TE-13 and Eca109 $48 \mathrm{~h}$ after transfection. (A) Knockdown of HO-1 in TE-13 cells was associated with increased MFI of intracellular ROS $\left({ }^{* *} \mathrm{P}<0.001\right.$, compared with the scramble). (B) Knockdown of HO-1 in Eca109 cells was associated with increased MFI of intracellular ROS $\left({ }^{* *} \mathrm{P}<0.001\right.$, compared with the scramble). 
A

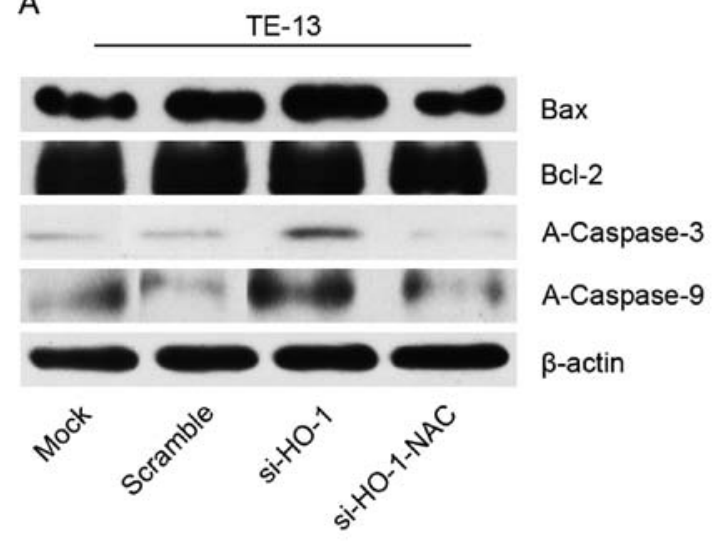

B

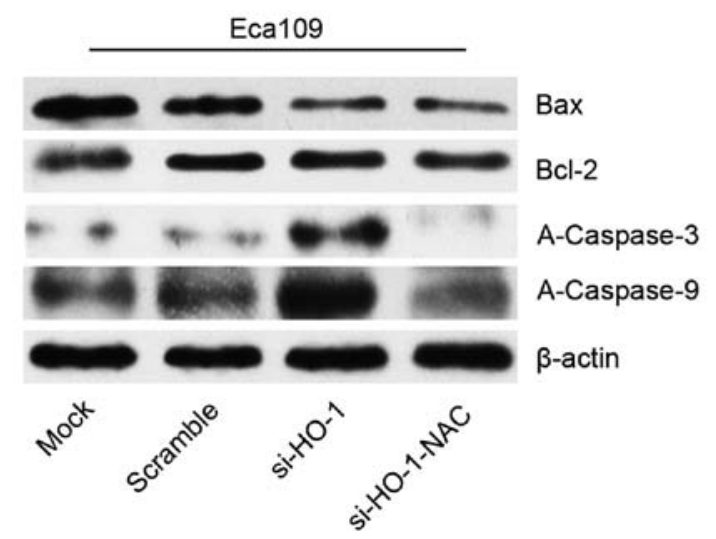

Figure 6. Effect of blocking HO-1 expression on the cell line by western blot expression of Bax, Bcl-2, A-caspase-3 and -9 at $48 \mathrm{~h}$ after transfection. (A) Expression of Bax, Bcl-2, A-caspase-3 and -9 in TE-13 cells before and after addition of $\mathrm{N}$-acetyl cysteine (NAC). There was significantly less expression after treatment with NAC ( $\mathrm{P}<0.05)$. (B) Expression of Bax, Bcl-2, A-caspase-3 and -9 in Eca109 cells before and after addition of NAC. There was significantly less expression after treatment with NAC ("P<0.05).

\section{Discussion}

HO-1 is an enzyme with strong antioxidant activity. The products of HO-1, biliverdin, carbon monoxide and ferrous iron, also have various antioxidant properties. HO-1 thus plays a vital role in the antioxidant system (8). Increased HO-1 expression has been observed in breast, gastric and lung cancer. Moreover, it has been reported that overexpression of HO-1 could inhibit tumor cellular apoptosis and promote tumor cell growth (9-11).

We found that HO-1, HIF-1 $\alpha$ and EGFR were expressed in $40.6 \%, 43.4 \%$ and $58 \%$, respectively, of 143 ESCC tumors. We also found that HO-1 expression was related to tumor grade, but not related to clinical stage or the presence of mediastinal lymph node metastases. These findings suggest HO-1 may have a role in ESCC progression.

As in general, hypoxia exists in cancer microenvironment also in ESCC. Under hypoxia condition, HIF-1 $\alpha$ acting as a key transcription factor is highly expressed and oxygen-free radicals accumulate. Evidence has shown that the expression of HO-1 was increased by HIF-1 $\alpha(12,13)$. Moreover, oxidation stress exists in tumors (14). Additionally, we found that HO-1 is positively correlated with HIF-1 $\alpha$. As a result, we inferred that HIF-1 $\alpha$ prompted the expression of $\mathrm{HO}-1$, and that the oxidation stress resulted from hypoxia also contributed to the increased expression of HO-1. High expression of EGFR can suppress tumor cell apoptosis and promote angiogenesis, proliferation and metastasis of tumor cells. EGFR could induce expression of HO-1 in colon cancer; EGFR-induced colon cancer cell proliferation was inhibited by the decreased expression of HO-1 (15). Thus, we deduced that EFGR could stimulate the expression of HO-1, as well. Taken together, we concluded that the expression of HIF-1 $\alpha$, EGFR and HO-1 were rather high and the expression of HO-1 was induced by HIF- $1 \alpha$ and EGFR in ESCC tumor progression.

We previously reported that ethanol increased HO-1 expression and decreased apoptosis in ESCC cells (6). In the present study, we demonstrated that decreasing HO-1 expression in ESCC cell lines decreased cellular proliferation and increased apoptosis. We then investigated whether this finding was associated with intracellular ROS levels or activation of apoptosis signaling pathways. Decreasing cell line HO-1 expression was found to be associated with increased intracellular ROS levels. Lin et al have reported that decreased HO-1 expression in renal carcinoma cells was associated with increased intracellular ROS production and that this was associated with damage to cellular DNA (16). ROS is a major product of oxidation-reduction reactions in human cells. Various ROS are always found in normally functioning cells and are thought to be a normal part of cell growth, proliferation and differentiation. ROS are usually found in very high levels in tumor cells (14). These high intracellular levels could affect normal cellular function and may contribute to tumor progression $(17,18)$

We hypothesized that the decrease in $\mathrm{HO}-1$ expression and ROS levels may affect the expression of apoptosis-related proteins. Bcl-2 and Bax are two important components of the mitochondrial apoptotic pathway. Bcl-2 inhibits the release of cytochrome $c$ from mitochondria to cytosol, which plays a vital role in inhibiting apoptosis. Bax has an opposing effect on the action of Bcl-2. ROS activates the mitochondrial apoptotic pathway by increasing Bax expression and decreasing Bcl-2 expression. Hambright et al have reported that NAC, a specific ROS scavenger, could inhibit the upregulation of Bax and downregulation of $\mathrm{Bcl}-2$ by altering ROS levels in melanoma cells (19). However, we found no significant changes in Bax or $\mathrm{Bcl}-2$ expression, or in the $\mathrm{Bax} / \mathrm{Bcl}-2$ expression ratio in ESCC cell lines we examined.

The powerful antioxidant NAC was used to scavenge ROS in our experiment. Treatment with NAC after transfection with si-HO-1 was associated with a significant decrease in MFI, increased cellular proliferation, and decreased apoptosis. These findings support a relationship between HO-1 expression and these events.

ROS are largely generated in mitochondria. The mitochondrial apoptosis pathway is a major apoptotic pathway that acts through the production of ROS. Caspases are responsible for the deliberate disassembly of cells into apoptotic bodies during apoptosis. Caspases-3,-8 and -9 appear to be regulators of this process. Caspase- 9 activates disassembly in response to events that trigger the release of cytochrome $c$ from mitochondria. Caspase-3 activity appears to regulate the speed of this response. The study of caspase $-3^{-/-}$and caspase- $-9^{-/-}$mice 
suggested the caspase pathway used for disassembly is cell type specific (20-22). Our findings suggest that control of HO-1 expression contributes to this process.

si-HO-1 transfected cell lines examined by us had significantly greater expression of A-caspase-3 and -9 than controls. Treatment of these cell lines with NAC, significantly decreased the expression of A-caspase-3 and -9. These findings suggest the mitochondrial apoptosis pathway may not be the only mechanism of controlling ROS-mediated apoptosis and supports the role of $\mathrm{HO}-1$ as a mediator of this alternate pathway. Choi et al reported that ROS can impact the expression of Fas, Fas-L and caspases-3, -8 and -9 in gastric cancer cells (23). This finding further supports the presence of a ROS-mediated non-mitochondrial pathway for cellular apoptosis.

In summary, we found increased tissue expression of HO-1 in ESCC. This expression was correlated with tumor grade and expression of EGFR and HIF-1 $\alpha$. Blocking HO-1 expression in ESCC cell lines resulted in decreased cellular proliferation, increased ROS levels, and increased cellular apoptosis. HO-1 appears to have a role in tumor progression via a mitochondrion-independent pathway.

\section{Acknowledgements}

The present study was supported by a grant from the Hubei Province Natural Science Foundation (grant no. 2014CFB390) and Wuhan Municipal Science and Technology Bureau (grant no. 2013060602010238).

\section{References}

1. Zeng H, Zheng R, Guo Y, Zhang S, Zou X, Wang N, Zhang L, Tang J, Chen J, Wei K, et al: Cancer survival in China, 2003-2005: A population-based study. Int J Cancer 136: 1921-1930, 2015.

2. Heasman SA, Zaitseva L, Bowles KM, Rushworth SA and Macewan DJ: Protection of acute myeloid leukaemia cells from apoptosis induced by front-line chemotherapeutics is mediated by haem oxygenase-1. Oncotarget 2: 658-668, 2011.

3. Tibullo D, Barbagallo I, Giallongo C, La Cava P, Parrinello N, Vanella L, Stagno F, Palumbo GA, Li Volti G and Di Raimondo F: Nuclear translocation of heme oxygenase-1 confers resistance to imatinib in chronic myeloid leukemia cells. Curr Pharm Des 19: 2765-2770, 2013.

4. Na HK and Surh YJ: Oncogenic potential of Nrf2 and its principal target protein heme oxygenase-1. Free Radic Biol Med 67: 353-365, 2014.

5. Wegiel B, Nemeth Z, Correa-Costa M, Bulmer AC and Otterbein LE: Heme oxygenase-1: A metabolic nike. Antioxid Redox Signal 20: 1709-1722, 2014.

6. Hu JL, Xiao L, Li ZY, Wang Q, Chang Y and Jin Y: Upregulation of HO-1 is accompanied by activation of p38MAPK and mTOR in human oesophageal squamous carcinoma cells. Cell Biol Int 37: 584-592, 2013.

7. Yang SL, Liu LP, Jiang JX, Xiong ZF, He QJ and Wu C: The correlation of expression levels of HIF-1 $\alpha$ and HIF-2 $\alpha$ in hepatocellular carcinoma with capsular invasion, portal vein tumor thrombi and patients' clinical outcome. Jpn J Clin Oncol 44: 159-167, 2014.
8. Wang S, Hannafon BN, Wolf RF, Zhou J, Avery JE, Wu J, Lind SE and Ding WQ: Characterization of docosahexaenoic acid (DHA)-induced heme oxygenase-1 (HO-1) expression in human cancer cells: The importance of enhanced BTB and CNC homology 1 (Bach1) degradation. J Nutr Biochem 25: 515-525, 2014.

9. Noh SJ, Bae JS, Jamiyandorj U, Park HS, Kwon KS, Jung SH, Youn HJ, Lee H, Park BH, Chung MJ, et al: Expression of nerve growth factor and heme oxygenase-1 predict poor survival of breast carcinoma patients. BMC Cancer 13: 516, 2013.

10. Yin Y, Liu Q, Wang B, Chen G, Xu L and Zhou H: Expression and function of heme oxygenase-1 in human gastric cancer. Exp Biol Med 237: 362-371, 2012.

11. Degese MS, Mendizabal JE, Gandini NA, Gutkind JS, Molinolo A, Hewitt SM, Curino AC, Coso OA and Facchinetti MM: Expression of heme oxygenase-1 in non-small cell lung cancer (NSCLC) and its correlation with clinical data. Lung Cancer 77: 168-175, 2012.

12. Miyake M, Fujimoto K, Anai S, Ohnishi S, Kuwada M, Nakai Y, Inoue T, Matsumura Y, Tomioka A, Ikeda T, et al: Heme oxygenase-1 promotes angiogenesis in urothelial carcinoma of the urinary bladder. Oncol Rep 25: 653-660, 2011.

13. Ben Mosbah I, Mouchel Y, Pajaud J, Ribault C, Lucas C, Laurent A, Boudjema K, Morel F, Corlu A and Compagnon P: Pretreatment with mangafodipir improves liver graft tolerance to ischemia/reperfusion injury in rat. PLoS One 7: e50235, 2012.

14. Chen W, Balakrishnan K, Kuang Y, Han Y, Fu M, Gandhi V and Peng X: Reactive oxygen species (ROS) inducible DNA cross-linking agents and their effect on cancer cells and normal lymphocytes. J Med Chem 57: 4498-4510, 2014.

15. Lien GS, Wu MS, Bien MY, Chen $\mathrm{CH}$, Lin $\mathrm{CH}$ and Chen BC: Epidermal growth factor stimulates nuclear factor- $\kappa \mathrm{B}$ activation and heme oxygenase-1 expression via c-Src, NADPH oxidase, PI3K, and Akt in human colon cancer cells. PLoS One 9: e104891, 2014.

16. Lin PH, Lan WM and Chau LY: TRC8 suppresses tumorigenesis through targeting heme oxygenase-1 for ubiquitination and degradation. Oncogene 32: 2325-2334, 2013.

17. Trachootham $D$, Alexandre $J$ and Huang P: Targeting cancer cells by ROS-mediated mechanisms: A radical therapeutic approach? Nat Rev Drug Discov 8: 579-591, 2009.

18. Sanchez-Alvarez R, Martinez-Outschoorn UE, Lin Z, Lamb R, Hulit J, Howell A, Sotgia F, Rubin E and Lisanti MP: Ethanol exposure induces the cancer-associated fibroblast phenotype and lethal tumor metabolism: Implications for breast cancer prevention. Cell Cycle 12: 289-301, 2013.

19. Hambright HG, Meng P, Kumar AP and Ghosh R: Inhibition of PI3K/AKT/mTOR axis disrupts oxidative stress-mediated survival of melanoma cells. Oncotarget 6: 7195-7208, 2015.

20. Josefsson EC, Burnett DL, Lebois M, Debrincat MA, White MJ, Henley KJ, Lane RM, Moujalled D, Preston SP, O'Reilly LA, et al: Platelet production proceeds independently of the intrinsic and extrinsic apoptosis pathways. Nat Commun 5: 3455, 2014.

21. Oh BS, Shin EA, Jung JH, Jung DB, Kim B, Shim BS, Yazdi MC, Iranshahi $\mathrm{M}$ and Kim SH: Apoptotic effect of galbanic acid via activation of caspases and inhibition of Mcl-1 in H460 non-small lung carcinoma cells. Phytother Res 29: 844-849, 2015.

22. Jiang L, Li L, He X, Yi Q, He B, Cao J, Pan W and Gu Z: Overcoming drug-resistant lung cancer by paclitaxel loaded dual-functional liposomes with mitochondria targeting and pH-response. Biomaterials 52: 126-139, 2015.

23. Choi YH, Kang YJ, Kim SH, Sung B, Kim DH, Hwang SY, Kim M, Lim HS, Yoon JH, Moon HR, et al: MHY-449 induces apoptotic cell death through ROS- and caspase-dependent pathways in AGS human gastric cancer cells. Cancer Res 75 (Suppl 15): S1773, 2015. doi: 10.1158/1538-7445.AM2015-1773. 